\title{
Investigation of the Dissolution Characteristics of Nifedipine Extended-Release Formulations Using USP Apparatus 2 and a Novel Dissolution Apparatus
}

\author{
Grzegorz Garbacz ${ }^{1,3}$, Henning Blume ${ }^{2}$, and Werner Weitschies ${ }^{1}$ \\ 'Department of Biopharmaceutics and Pharmaceutical Technology, Ernst Moritz Arndt \\ University of Greifswald, Friedrich-Ludwig-Jahn-Strasse 17, D-17487 Greifswald, Germany \\ ${ }^{2}$ SocraTec R\&D GmbH, Oberursel, Germany
}

\begin{abstract}
The aim of our studies was to investigate the dissolution characteristics of six extended-release nifedipine tablets with a dosage strength of $20 \mathrm{mg}$ that are marketed in the European Union. All investigated products were homogenous matrix tablets having identical dosing regimens. The products can be substituted for originator products and other ER generic formulations of the same drug load. In the present study, the formulations were tested using USP Apparatus 2 at 50 rpm, which is one of the standard conditions specified by the USP for nifedipine ER tablets. Moreover, investigations under intensified stress conditions were performed with Apparatus 2 at 100 rpm, as well as in a novel biorelevant dissolution stress-test device. A complementary characterization of the integrity of the tablet matrices was performed using a standard disintegration test apparatus. In the applied experimental settings, drug dissolution from all tested formulations proceeded very fast with liberation of at least $15 \mathrm{mg}$ of drug within the first two hours of analysis. The increase in stirring rate from 50 to $100 \mathrm{rpm}$ in Apparatus 2 did not substantially accelerate the dissolution process. Moreover, it was noticed that the tablet matrices had poor mechanical stability and very rapid disintegration behavior under all experimental settings. The disintegration times measured in the standard disintegration test were less than 10 min. The results clearly show that the dissolution characteristics of all tested products are a function of the properties of the drug substance and not the dosage forms. It became obvious that none of the tested formulations fulfilled USP requirements for nifedipine ER tablets specified for Apparatus 2 at $50 \mathrm{rpm}$. However, the products are marketed in the European Union where the USP quality criteria are not mandatory but only advisable. In our interpretation, such dissolution characteristics bear a potential risk of dosage-form-related interactions in vivo.
\end{abstract}

\section{INTRODUCTION}

$\mathrm{N}$ ifedipine is a calcium-channel blocking agent that is widely used in the treatment of angina pectoris and systemic hypertension (1). The pharmacokinetics and pharmacodynamics of nifedipine have been investigated in numerous studies (2-4).

Clinical experiences gained with oral nifedipine formulations with immediate-release (IR) characteristics clearly show that a steep rise in the drug plasma concentration results in an increase in heart rate and drug-specific side effects (5-7). Therefore, it has been generally accepted that extended-release (ER) formulations are most efficient for routine hypertension therapy with nifedipine. The ER dosage forms should primarily reduce the occurrence of steep rises in plasma concentration of the drug. Another important therapeutic goal that can be achieved with ER formulations is the improvement of chronic therapy compliance by prolongation of the dosing intervals.

${ }^{3}$ Corresponding author.
The pharmacokinetics of orally administrated modifiedrelease nifedipine has been investigated in several clinical studies. In most cases, irregular concentration-time profiles were observed, especially when the dosage forms were given with a meal (8-10). A pharmacokinetic interaction at the absorption site has been discussed as an explanation for the observed food effect. Another possible explanation is the lack of robustness of the dosage form under in vivo conditions with respect to the well-known high variability of the physiological $\mathrm{pH}$ values and the composition of gastrointestinal dissolution media $(11,12)$. Less attention has been paid to the mechanical robustness of the dosage form despite the impact of physiological mechanical stress. To date, this aspect is poorly understood and has been studied only sketchily in some rather simplified experiments (13). However, in vivo data characterizing the main physiological stress factors that may affect drug dissolution from a solid dosage form during the gastrointestinal (Gl) passage have become increasingly available, and a biorelevant dissolution stress-test apparatus has recently been developed (14). This device simulates three physical conditions that dosage forms 
experience during gastrointestinal transport and that may affect the drug dissolution process. Using this device, the physiological movement of the dosage form can be simulated. Furthermore, the stress-test apparatus offers the possibility of simulating the agitation caused by gastrointestinal pressure waves. Finally, an interrupted contact of the dosage form to the liquid medium can be simulated.

\section{MATERIALS AND METHODS \\ Dosage Forms}

In this study, six ER tablet formulations, each containing $20 \mathrm{mg}$ nifedipine, were examined. All tablets were homogenous matrix tablets that are marketed in the European Union and have identical dosing regimens, which is twice a day. The products can be substituted for originator products and other ER generic formulations of the same drug load. The compositions of the tested formulations are given in Table 1.

\section{Applied Test Media}

All dissolution experiments were carried out in USP pH 6.8 buffer for nifedipine ER tablets with 1\% SDS $(84 \mathrm{mOsm} / \mathrm{kg})$ as the dissolution medium. For the determination of the disintegration behavior of the dosage forms, purified water was used as a complementary medium.

\section{Standard Dissolution Tests}

The dissolution characteristics of the tablets were examined using USP Apparatus 2 (paddle apparatus,

\section{Table 1. Composition of the Tested Formulations}

\begin{tabular}{ll}
\hline Formulation No. & \multicolumn{1}{c}{ Excipients } \\
\hline 1 & $\begin{array}{l}\text { iron oxide red, lactose monohydrate, macrogol 4000, } \\
\text { magnesium stearate, corn starch, hydroxypropyl } \\
\text { methylcellulose, microcrystalline cellulose, } \\
\text { polysorbate 80, titanium dioxide }\end{array}$ \\
\hline 2 & $\begin{array}{l}\text { microcrystalline cellulose, macrogol, magnesium } \\
\text { stearate, mannitol, hydroxypropyl methylcellulose, } \\
\text { sodium dodecyl sulfate, carboxymethyl starch sodium, } \\
\text { povidone K 25, silicon dioxide, talc, titanium dioxide, } \\
\text { iron oxide red }\end{array}$ \\
\hline 3 & $\begin{array}{l}\text { lactose monohydrate, corn starch, microcrystalline } \\
\text { cellulose, polysorbate } 80, \text { magnesium stearate, } \\
\text { hydroxypropyl methylcellulose, macrogol 4000, iron } \\
\text { oxide red }\end{array}$ \\
\hline 4 & $\begin{array}{l}\text { cellulose, hydroxypropyl methylcellulose, lactose, } \\
\text { macrogol 4000, magnesium stearate, corn starch, } \\
\text { polysorbate 80, iron oxide red }\end{array}$ \\
\hline 5 & $\begin{array}{l}\text { talc, microcrystalline cellulose, lactose, croscarmellose } \\
\text { sodium, stearic acid, silicium dioxide, magnesium } \\
\text { stearate, hydroxypropyl methylcellulose, macrogol, } \\
\text { iron oxide red }\end{array}$ \\
\hline $\begin{array}{l}\text { microcrystalline cellulose, lactose monohydrate, } \\
\text { macrogol 6000, magnesium stearate, corn starch, } \\
\text { hydroxypropyl methylcellulose, polysorbate 80, talc, } \\
\text { iron oxide red, titanium dioxide }\end{array}$ \\
\hline
\end{tabular}

Dissolution Technologies | MAY 2009
PT-DT 7, PharmaTest, Hainburg, Germany) at rotational speeds of $50 \mathrm{rpm}$ and $100 \mathrm{rpm}$ at $37.0 \pm 0.5^{\circ} \mathrm{C}$. This device was chosen as one of the most popular and wellestablished test methods for the quality control of solid oral dosage forms. The amount of drug dissolved was determined using a spectrophotometer (UV 1650, Shimadzu, Germany) equipped with a multi-cell positioned by a closed flow-through system. A peristaltic pump (IPC-N 16, Ismatec, Germany) circulated dissolution medium in the system at a flow rate of $10 \mathrm{~mL} / \mathrm{min}$ in a pump interval of five minutes duration (three minutes of pumping and two minutes of rest), while filtering the medium through a glass filter ( $20 \mu \mathrm{m}$, Jena Glas, Germany) under isothermal conditions. The filtrate was pumped through a quartz flow-through cell (10-mm path, Hellma, Germany).The absorption was measured at 5-min intervals in a differential mode at $350 \mathrm{~nm}$ (signal) and $450 \mathrm{~nm}$ (background) and calculated as the differential value. Data acquisition was performed with commercial software (UV-Probe, Shimadzu, Germany). To avoid photodegradation of nifedipine, all analytical operations and tests were carried out with artificial red light of $\lambda \geq 500 \mathrm{~nm}$ (LED Spotlight WEC Cooperatios 18 LEDs, 230 V / 1,5 W, JDR E 27) as the sole laboratory illumination.

\section{Dissolution Stress Test}

The dissolution stress-test device enables the exposure of the dosage forms to an arbitrary sequence of movements, pressure waves, and phases of rest that may occur under in vivo conditions. The device consists of a steel central pipe (axis) with six steel netting spheres (chambers) of 35-mm diameter in which the dosage forms are placed. Each chamber is divided into two parts. The bottom part is screwed onto the central pipe by a PVC bushing and a profiled nozzle. The central pipe is attached by Teflon handles placed on the deck plate of the device very closely $(3 \mathrm{~mm})$ above the top edges of standard dissolution vessels placed linearly in their symmetry plane. Each sphere operates in a separate vessel. On one end, the central axis is coupled with a pressure regulation unit by a rotating joint, and on the other end with a stepping motor. The schematic and photographic illustration of the test device is given in Figure 1.

Pressure waves are generated by pulsatile inflation and deflation of balloons inside the chambers. The process is controlled by the synchronized switching of solenoid valves (ASCO G262C022, ASCO Jucomatic, Germany). The pressure is regulated by a computer-controlled pressure-reducing device (Norgren R16-200-R30D, Germany). The central axis is driven by a computercontrolled stepping motor (St 5818M1008, Optocoppler SMC 32C, Nanotec, Germany). The test parameters are software-controlled (LabView 7.1, National Instruments, USA).

All experiments with the stress-test device were carried out in $1150 \mathrm{~mL}$ of dissolution medium. This volume 


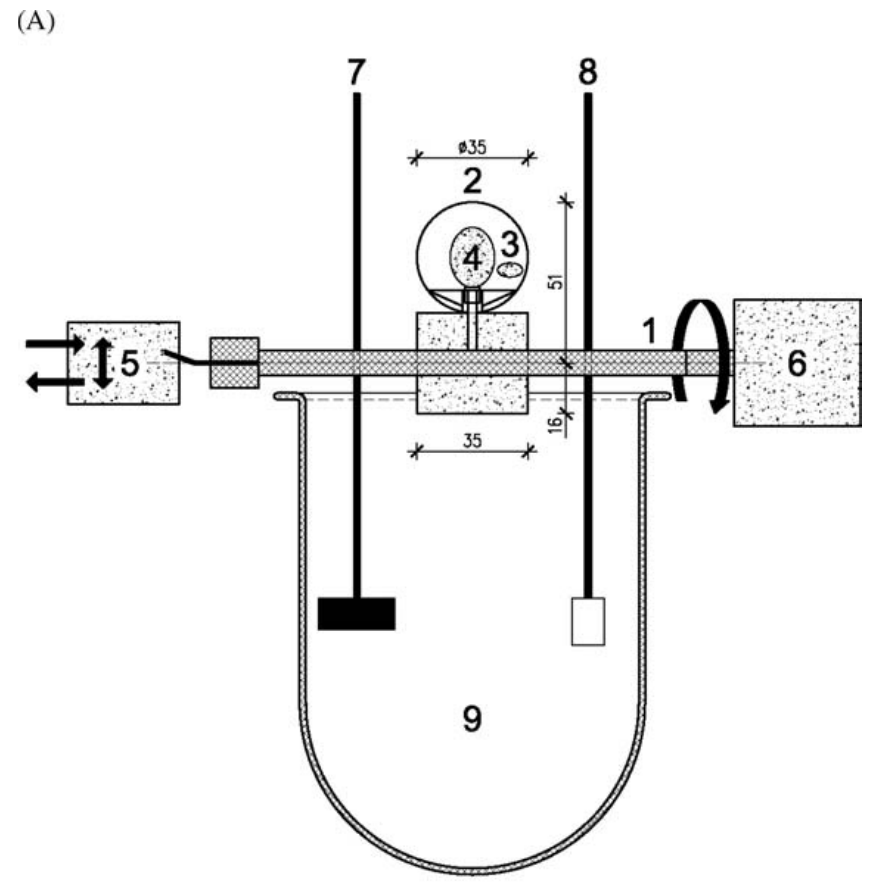

(B)

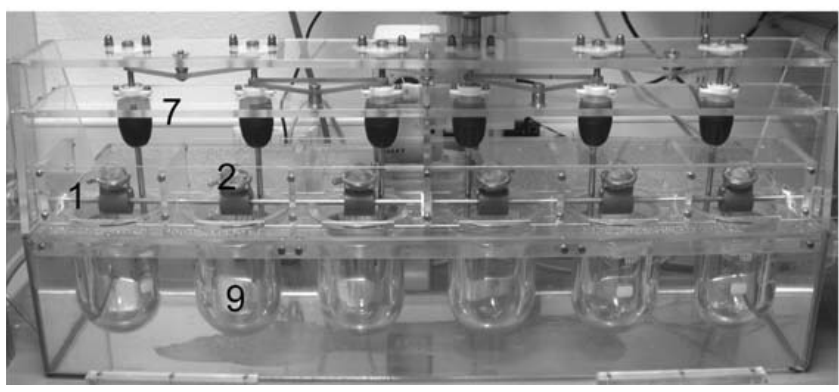

Figure 1. (A) Schematic and (B) photographic representation of the biorelevant dissolution stress-test device: 1-central axle (Ø8 mm), 2-steel netting wire chamber ( $\varnothing 35 \mathrm{~mm}$, mesh size $0.5 \mathrm{~mm}$, wire $0.1 \mathrm{~mm}$ ), 3-dosage form, 4-inflatable balloon, 5-solenoid valve system, 6-stepping motor, 7-stirrer (blade $35 \times 15 \mathrm{~mm}$ ), 8-sampling, 9-standard dissolution vessel.

assured the complete immersion of the steel wire netting spheres in the dissolution medium during the phases of rest when the spheres were placed vertically down. The medium was homogenized by a steel paddle stirrer (15 $\mathrm{mm} \times 35 \mathrm{~mm}$ stirrer blade) that was operated at $100 \mathrm{rpm}$.

The device was driven in a test program that mimics the physiological stress transport phenomena as they are observed in vivo. The applied program is a combination of high and low agitation phases as well as phases of rest. The immersion of the dosage forms in the dissolution medium was defined as the starting point of the analysis. This was achieved by positioning the apparatus axis in such way that the steel wire netting spheres were angled vertically down. The high-stress phase simulated at $0.5 \mathrm{~h}$ consisted of a sequence of three symmetrical pressure waves of 300 mbar with a duration of $6 \mathrm{~s}$ each, followed by a 1-min rotation phase at a stirring speed of $100 \mathrm{rpm}$. This phase simulated high mechanical stress acting on the dosage form during passage through high motor activity zones of the $\mathrm{Gl}$ tract like the pyloric or ileocecal regions. The low-agitation phase simulated after one, two, and four hours was composed of a 1-min rotational movement phase at $60 \mathrm{rpm}$ mimicking the mild transport phenomenon observed during passage through the small intestine. Before the first stress phase and between the subsequent stress phases, the probe chambers were submersed in the dissolution medium, and no motion of the apparatus axis was stimulated.

The amount of drug dissolved was determined using a UV-vis spectrophotometer (Cary 50, Varian Instruments) equipped with a fiber-optic device with a 10-mm light path and 1-s signal averaging time. The measurement proceeded in a differential mode at $350 \mathrm{~nm}$ (signal) and $450 \mathrm{~nm}$ (disturbances). The effective absorbance $(A)$ was calculated according to the equation $A=A_{350}-A_{450}$.

\section{Tablet Disintegration Test}

The disintegration of nifedipine ER tablets was tested in USP buffer for nifedipine ER tablets and purified water using a standard disintegration apparatus (Ph. Eur. Apparatus A) at $37 \pm 0.5^{\circ} \mathrm{C}$ and 30 dips per minute. The determination of the end point of tablet disintegration was performed visually and was expressed as the time of disintegration of the last of the six tested dosage forms.

\section{RESULTS}

\section{Standard Dissolution Test}

The dissolution profiles obtained with USP Apparatus 2 operated at rotational speeds of $50 \mathrm{rpm}$ and $100 \mathrm{rpm}$ are presented in Figure 2. At a stir rate of 50 rpm, nifedipine release was very fast, and at least $10 \mathrm{mg}$ dissolved within the first $30 \mathrm{~min}$ for all formulations. For Formulations 2 and $5,10 \mathrm{mg}$ of drug was released after only $10 \mathrm{~min}$. The time required for dissolution of $15 \mathrm{mg}$ ranged from $25 \mathrm{~min}$ for Formulations 2 and 6 , to about $1.2 \mathrm{~h}$ for Formulations 1 and 3 . The increase in stirring rate from 50 to $100 \mathrm{rpm}$ resulted in a further acceleration of the dissolution rates only for Formulations 4 and 5, while no meaningful differences in the dissolution profiles of the other formulations were observed.

During the dissolution experiments, rapid disintegration of the tablet matrices was observed for all tested products.

\section{Dissolution Stress Test}

The dissolution profiles obtained using the biorelevant dissolution stress-test apparatus are presented in Figure 3. Nifedipine dissolution was fastest for Formulation 2, with approximately $1 \mathrm{~h}$ required for complete dissolution, and was slowest for Formulation 4, with about $5 \mathrm{~h}$ required for complete dissolution. 

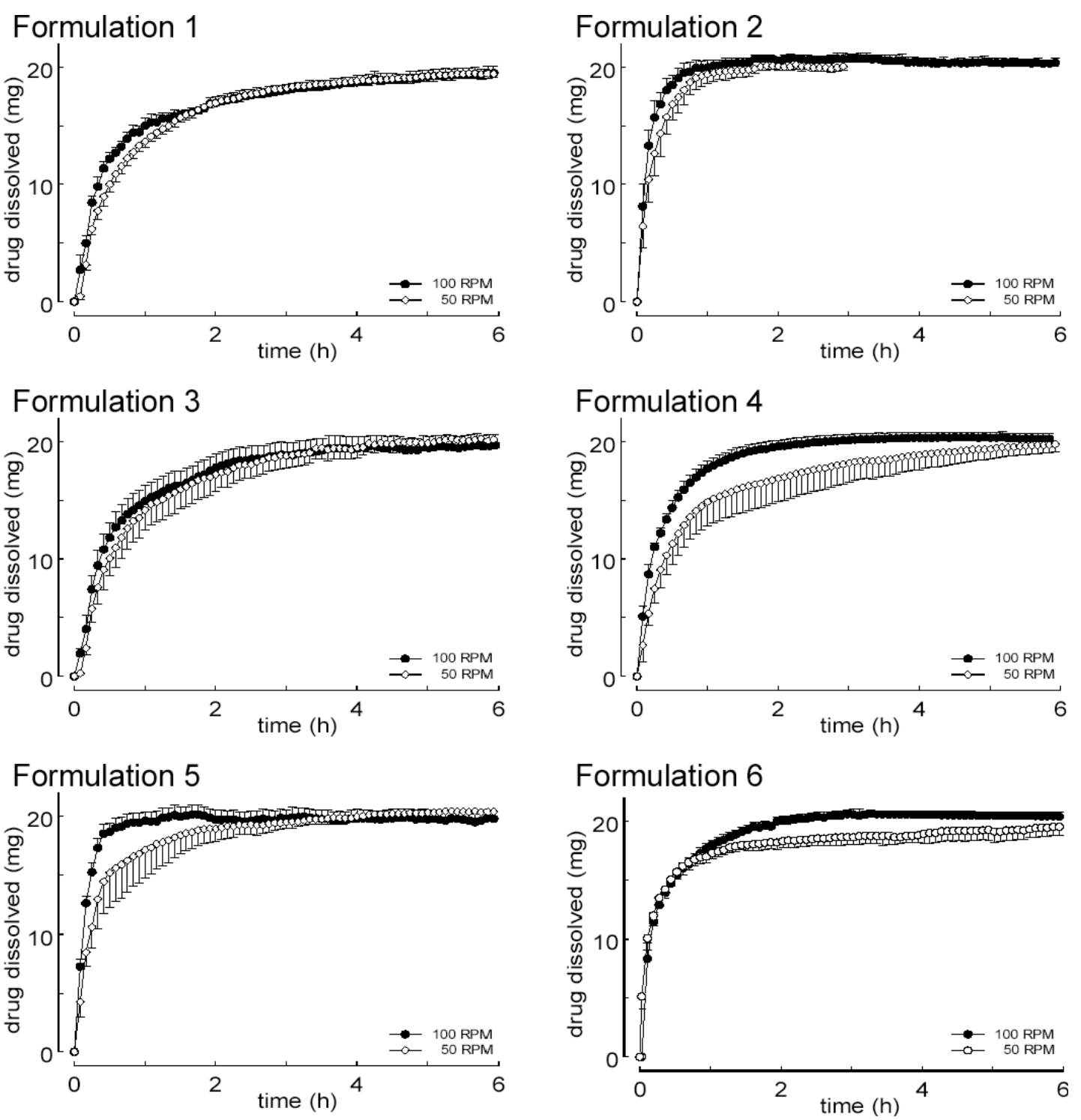

Figure 2. Drug dissolution profiles obtained using USP Apparatus 2 in USP pH 6.8 buffer for nifedipine ER tablets, $900-\mathrm{mL}$ fill volume, $37^{\circ} \mathrm{C}$, rotational speeds of $100 \mathrm{rpm}(\bullet)$ and $50 \mathrm{rpm}(\circ)$. Mean values $(n=6)$ are shown, standard deviations are given by the error bars.

For Formulations 2 and 6 , use of the stress conditions did not influence the dissolution characteristics of the tablets because in both cases, the disintegration process was completed before the first agitation phase. The nifedipine dissolution proceeded at a rate that was comparable to those obtained with USP Apparatus 2 at $50 \mathrm{rpm}$. The first stress phase applied at $0.5 \mathrm{~h}$ had the most remarkable impact on the dissolution profiles of Formulations $1,3,4$, and 5 , with a rapid release of approximately $6 \mathrm{mg}$ nifedipine from Formulation 1 and $10 \mathrm{mg}$ from Formulation 4. A visual inspection of the probe chambers of the dissolution stress-test apparatus carried out immediately after application of the first stress phase showed that Formulations 3-5 disintegrated during this stress phase. Therefore, the later stress phases did not produce distinct increases ( $\geq 4 \mathrm{mg} / 5 \mathrm{~min}$ ) in the amount of drug dissolved. In Formulation 1, the phase of rotational movement at $60 \mathrm{rpm}$ simulated at $1 \mathrm{~h}$ resulted in the disintegration of the tablets and rapid dissolution of about $4 \mathrm{mg}$ of nifedipine. Because of complete disintegration of the matrices, the stress phases that followed at 2 and $4 \mathrm{~h}$ had no relevant impact on the dissolution characteristics of this formulation.

\section{Tablet Disintegration}

The susceptibility of the tablets to mechanical agitation was investigated with the standard disintegration test using pH 6.8 USP buffer for nifedipine ER tablets and purified water as a reference medium of distinctly higher surface tension. The observed disintegration behavior of 


\section{Formulation 1}

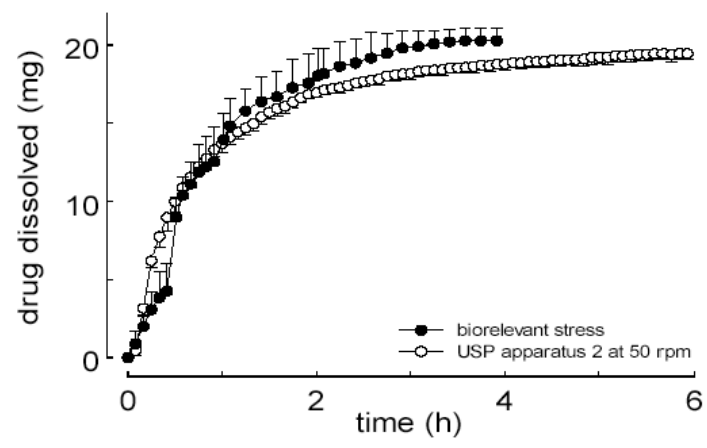

Formulation 3

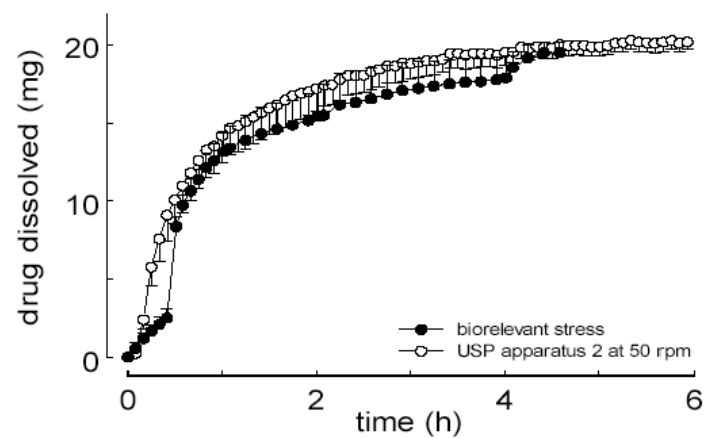

Formulation 5

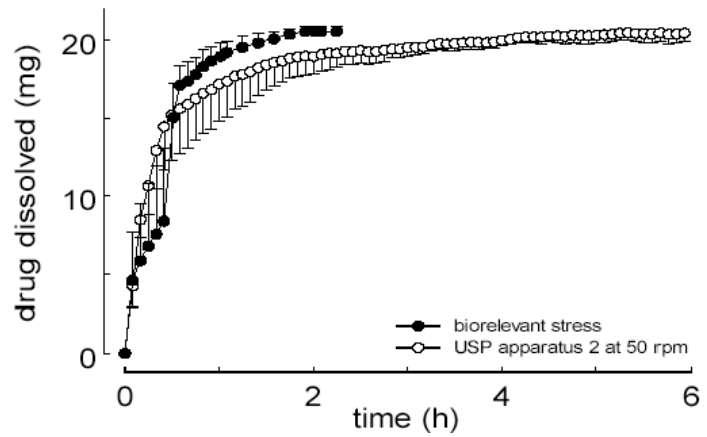

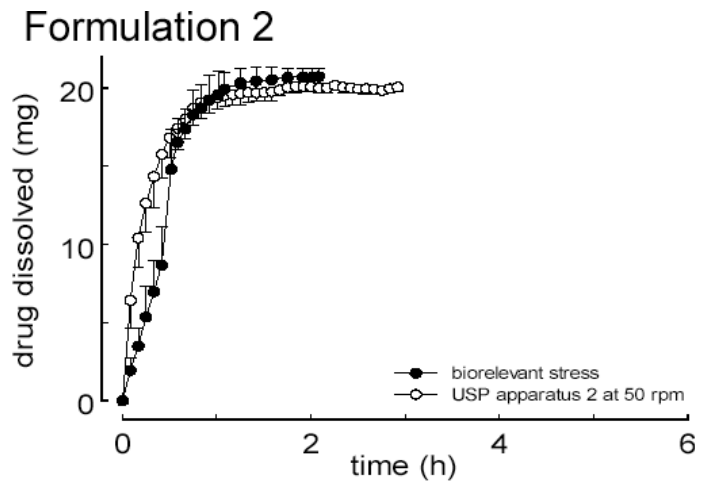

Formulation 4

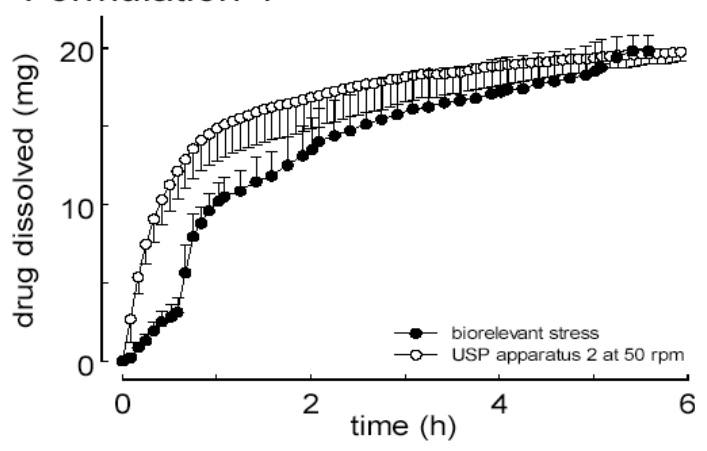

Formulation 6

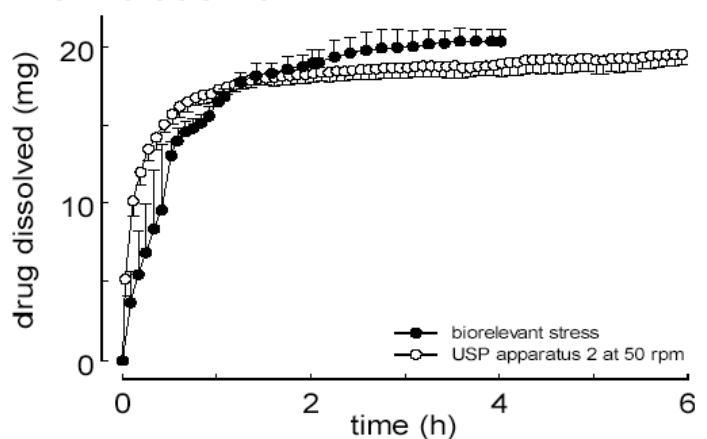

Figure 3. Drug dissolution profiles obtained using the biorelevant stress-test apparatus $(\bullet)$ (USP pH 6.8 buffer for nifedipine ER tablets, $1150-\mathrm{mL}$ fill volume, $37^{\circ} \mathrm{C}$ ) and USP Apparatus 2 at $50 \mathrm{rpm}$ ( $\left(\right.$ ) (USP pH 6.8 buffer for nifedipine ER tablets $900 \mathrm{~mL}$ fill volume, $\left.37^{\circ} \mathrm{C}\right)$. Presented are mean values $(n=6)$, standard deviations are shown by error bars.

the investigated products is presented in Table 2. Rapid disintegration of all tested formulations was observed with disintegration times below 10 min for all tested products, ranging from 0.33 min for Formulation 5 to $9.17 \mathrm{~min}$ for Formulation 3. The applied media had only minor influence on disintegration behavior. The obtained data are in good agreement with the results of visual inspection carried out during the dissolution experiments and confirm the observation of poor mechanical stability of the tested tablets under the applied test conditions.

Table 2. Summary of the Disintegration Tests Results

Disintegration Time (min)

Medium

Formulation $1 \quad$ Formulation $2 \quad$ Formulation $3 \quad$ Formulation 4

Formulation 5

Formulation 6

\begin{tabular}{lllllll}
\hline USP pH 6.8 nifedipine ER tablet buffer & 8.5 & 2.5 & 7.33 & 1.17 & 0.33 \\
\hline Purified water & 7.5 & 1.34 & 9.17 & 0.5 & 0.58 & 0.75 \\
\hline
\end{tabular}




\section{DISCUSSION}

According to the Biopharmaceutics Classification System (BCS), nifedipine is a typical Class II compound with low solubility and high permeability (15). Nifedipine is characterized by high absorption rates from the small intestine as well as the colon (16). Therefore, the plasma concentration-time profiles obtained after intake of an ER formulation of nifedipine should be determined mainly by the release characteristics of the dosage form and not the solubility kinetics of the drug substance.

The results of the present study clearly show that drug dissolution from all six tested formulations is not controlled by the properties of the tablet matrices but is determined by the dissolution characteristics of the nifedipine crystals that are released during the spontaneous disintegration of the tablet matrices. Such a strategy for the modification of the drug dissolution profiles of poorly water soluble drugs has been described previously (17) and bears a high risk of potential formulation-dependent interactions in vivo. At first, the in vivo dissolution characteristics of such preparations are strongly dependent on the liquid media to which the drug-containing particles are exposed during Gl transit. These are characterized by a high variability in their compositions and physicochemical properties. Furthermore, they are inhomogeneously distributed along the Gl lumen in pockets of variable volume $(12,18)$. However, due to the relatively small amount of water available in the lumen of the GI tract and the low solubility of nifedipine, a prolongation of the absorption process of the drug is likely, especially under fasted conditions. The results of the present study show that in all tested products, such an effect can be determined only by the character of the drug substance and not by the dissolution characteristic of tablets that are declared as extended-release dosage forms by the manufacturers.

Secondly, when such formulations are administered with food, it is very likely that the disintegration process of the dosage form will already be completed in the stomach. The drug-containing particles are usually inhomogeneously mixed with chyme, which can improve the drug solubility depending on its composition, whereas the systemic delivery of the drug is primarily controlled by the rate of gastric emptying $(19,20)$.

In previous works $(7,21-23)$, it has been shown that the kinetics of nifedipine delivery into systemic circulation is an important factor affecting drug response and the occurrence of side effects. Only a slow increase in drug plasma concentration results in the desired reduction of diastolic and systolic blood pressures.

Accordingly, dissolution requirements for nifedipine ER tablets were specified by the USP.The fraction of drug dissolved using USP Apparatus 2 at $50 \mathrm{rpm}$ is specified for nifedipine $20 \mathrm{mg}$ ER tablets as $10-30 \%$ (2-6 mg) release at $3 \mathrm{~h}, 40-65 \%(8-13 \mathrm{mg})$ at $6 \mathrm{~h}$, and not less than $80 \%$ (16 $\mathrm{mg}$ ) at $12 \mathrm{~h}(24)$. The study results demonstrate that none of the tested products fulfill the requirements of the USP.
However, the products are available on the European market where the quality criteria of the USP are advisable but not mandatory.

\section{CONCLUSIONS}

This study yielded differences in the pharmaceutical availability of nifedipine from tested formulations that are considered for administration in identical dosing intervals. The tested products can be substituted for originator and other ER generic formulations of the same drug load. However, the test results clearly demonstrate that the dissolution characteristics of all tested formulations is primarily controlled by the properties of the drug substance and not by the dosage forms. Considering the rapid dissolution characteristics and the susceptibility of the tablets to mechanical stress, drug delivery in vivo will likely proceed faster than would be clinically desirable.

\section{REFERENCES}

1. Schwartz, A.; Matlib, M. A.; Balwierczak, J.; Lathrop, D. A. Pharmacology of calcium antagonists. Am. J. Cardiol. 1985, 55 (7), 3C-7C.

2. Soons, P. A.; Schoemaker, H. C.; Cohen, A. F.; Breimer, D.D. Intraindividual variability in nifedipine pharmacokinetics and effects in healthy subjects. J. Clin. Pharmacol. 1992, 32, 324.

3. Walley, T.J.; Heagerty, A. M.;Woods, K. L.; Bing, R. F.; Pohl, J.E.; Barnett, D. B. Pharmacokinetics and pharmacodynamics of nifedipine infusion in normal volunteers. Brit. J.Clin. Pharmacol. 1987, 23 (6), 693-701.

4. Robertson, D. R.; Waller, D. G.; Renwick, A. G.; George, C. F. Age-related changes in the pharmacokinetics and pharmacodynamics of nifedipine. Brit. J. Clin. Pharmacol. 1988, 25 (3), 297-305.

5. Banzet, O.; Colin, J. N.; Thibonnier, M.; Singlas, E.; Alexandre, J. M.; Corvol, P. Acute antihypertensive effect and pharmacokinetics of a tablet preparation of nifedipine. Eur. J. Clin. Pharmacol. 1983, 24 (2), 145-50.

6. Toal, C.B. Formulation dependent pharmacokineticsdoes the dosage form matter for nifedipine? J. Cardiovasc. Pharm. 2004, 44 (1), 82-86.

7. Meredith, P. A.; Elliott, H. L. Dihydropyridine calcium channel blockers: basic pharmacological similarities but fundamental therapeutic differences. J. Hypertens. 2004, 22 (9), 1641-1648.

8. Schug, B.; Brendel, E.; Wolf, D.; Wonnemann, M.; Wargenau, M.; Blume, H. H. Formulation-dependent food effects demonstrated for nifedipine modifiedrelease preparations marketed in the European Union. Eur. J. Pharm. Sci. 2002, 15 (3), 279-285.

9. Wonnemann, M.; Schug, B.; Anschütz, M.; Brendel, E.; Nucci, G. D.; Blume, H. Comparison of two marketed nifedipine modified-release formulations: An exploratory clinical food interaction study. Clin. Ther. 2008, 30 (1), 48-58. 
10. Schug, B.; Brendel, E.; Chantraine, E.; Wolf, D.; Martin, W.; Schall, R.; Blume, H.H. The effect of food on the pharmacokinetics of nifedipine in two slow release formulations: pronounced lag-time after a high fat breakfast. Brit. J. Clin. Pharmacol. 2002, 53 (6), 582-588.

11. Russell, T. L.; Berardi, R. R.; Barnett, J.L.; Dermentzoglou, L.C.; Jarvenpaa, K. M.; Schmaltz, S. P.; Dressman, J. B. Upper Gastrointestinal pH in Seventy-Nine Healthy, Elderly, North American Men and Women. Pharm. Res. 1993, 10 (2), 187-196.

12. Jantratid, E.; Janssen, N.; Reppas, C.; Dressman, J. B. Dissolution Media Simulating Conditions in the Proximal Human Gastrointestinal Tract: An Update. Pharm. Res. 2008, (7), 1663-1676.

13. Qureshi, S. A.; Shabnam, J. Applications of a new device (spindle) for improved characterization of drug release (dissolution) of pharmaceutical products. Eur.J. Pharm. Sci. 2003, 19 (4), 291-297.

14. Garbacz, G.;Wedemeyer, R. S.; Nagel, S.; Giessmann, T.; Mönnikes, H.; Wilson, C. G.; Siegmund, W.; Weitschies, W. Irregular absorption profiles observed from diclofenac extended release tablets can be predicted using a dissolution test apparatus that mimics in vivo physical stresses. Eur. J. Pharm. Biopharm. 2008, 70 (2), 421-428.

15. Sutton, S. C.; Evans, L. A.; Fortner, J. H.; McCarthy, J. M.; Sweeney, K. Dog colonoscopy model for predicting human colon absorption. Pharm. Res. 2006, 23 (7), 1554-1563.

16. Bode, H.; Brendel, E.; Ahr, G.; Fuhr, U.; Harder, S.; Staib, A. H. Investigation of nifedipine absorption in different regions of the human gastrointestinal (GI) tract after simultaneous administration of ${ }^{13} \mathrm{C}$ - and ${ }^{12} \mathrm{C}$-nifedipine. Eur. J.Clin. Pharmacol. 1996, 50, 195-201.

17. Scholz, A.; Abrahamsson, B.; Diebold, M. S.; Kostewicz, E.; Polentarutti, B. I.; Ungell, A.-L.; Dressman, J. B. Influence of hydrodynamics and particle size on the absorption of felodipine in labradors. Pharm. Res. 2002, $19(1), 42-46$.

18. Schiller, C.; Froehlich, C.-P.; Giessman, T.; Siegmund, W.; Moennikes, H.; Hosten, N.; Weitschies, W. Intestinal fluid volumes and transit of dosage forms as assessed by magnetic resonance imaging. Aliment. Pharm. Therap. 2005, 22 (10), 971-979.

19. Lee, C.; Sarna, S. K. Central regulation of gastric emptying of solid nutrient meals by corticotropin releasing factor. Neurogastroent. Motil. 1997, 9, 221-229.

20. Marciani, L.; Gowland, P. A.; Spiller, R. C.; Manoj, P.; Moore, R. J.; Young, P.; Fillery-Travis, A. J. Effect of meal viscosity and nutrients on satiety, intragastric dilution, and emptying assessed by MRI.Am.J.Physiol.-Gastr. L. 2001,280, G1227-G1233.
21. Walley, T. J.; Heagerty, A. M.; Woods, K. L.; Bing, R. F.; Pohl, J.E.; Barnett, D. B. Acute inotropic effects of intravenous nifedipine and its vehicle compared with saline: a double-blind study of systolic time intervals in normal subjects. Brit. J. Clin. Pharmacol. 1988, 25 (2), 187-193.

22. Donnelly, R.; Elliott, H. L.; Meredith, P. A.; Kelman, A.W.; Reid, J. L. Nifedipine: individual responses and concentration-effect relationships. Hypertension 1988, $12,443-449$.

23. Kleinbloesem, C. H.; Van Brummelen, P.; Van de Linde, J. A.; Voogd, P. J.; Breimer, D. D. Nifedipine: kinetics and dynamics in healthy subjects. Clin. Pharmacol. Ther. 1984, 35 (6), 742-749.

24. Nifedipine Extended-Release Tablets Monograph. In United States Pharmacopeia and National Formulary USP 29-NF 24; The United States Pharmacopeial Convention, Inc.: Rockville, MD, 2006. 